\title{
Association of Mycoplasma pneumoniae infection with increased risk of asthma in children
}

\author{
SHA-SHA YIN, FENG-LIAN MA and XING GAO \\ Department of Pediatric Medicine, Linyi People's Hospital, Linyi, Shandong 276000, P.R. China
}

Received June 17, 2016; Accepted November 11, 2016

DOI: $10.3892 /$ etm.2017.4219

\begin{abstract}
The present study was conducted to investigate the relationship between Mycoplasma pneumoniae (MP) infection and the risk of asthma among children by detecting the rate of MP immunoglobulin M (MP-IgM) and the eosinophil (EOS) count. A total of 139 asthmatic children were enrolled as the case group and assigned into three groups: Group A (aged $<3$ years, $n=42$ ), group B (aged 3-8 years, $\mathrm{n}=45$ ) and group $\mathrm{C}$ (aged $>8$ years, $\mathrm{n}=52$ ). Additionally, 115 healthy children were enrolled in the control group. Enzyme-linked immunosorbent assay was used to measure the MP-IgM-positive rate. EOS count was detected in the experimental and control groups by using a hemocytometer analyzer. A meta-analysis was performed by using the Comprehensive Meta-Analysis version 2.0 software. The positive rates of the MP-IgM and EOS count in the experimental group were significantly higher than those in control group (both $\mathrm{P}<0.001$ ). Furthermore, the asthmatic children in group $\mathrm{C}$ had a higher MP-IgM-positive rate and EOS count as compared to those in groups $\mathrm{A}$ and $\mathrm{B}$, respectively (all $\mathrm{P}<0.05)$. Results from groups $\mathrm{A}$ and $\mathrm{B}$ were not statistically significant (all $\mathrm{P}>0.05$ ). The meta-analysis further confirmed that asthmatic children had a higher MP-IgM-positive rate as compared to the healthy controls $(\mathrm{P}<0.001)$. Age-stratified analysis revealed that the MP-IgM-positive rate in asthmatic children aged $\geq 8$ and $<8$ years was significantly higher than that in the healthy controls $(\mathrm{P}=0.003$ and $\mathrm{P}<0.001)$. Asthmatic children had a higher MP-IgM-positive rate and EOS count as compared with controls, suggesting that the MP infection may be closely associated with the risk of asthma. Additionally, the positive rate of MP-IgM may indicate an important biological marker in predicting the development of asthma.
\end{abstract}

Correspondence to: Dr Xing Gao, Department of Pediatric Medicine, Linyi People's Hospital, 27 Jiefang Road, Linyi, Shandong 276000, P.R. China

E-mail: yss20160421@163.com

Key words: Mycoplasma pneumoniae infection, asthma, case-control study, eosinophil count, meta-analysis, Mycoplasma pneumoniae immunoglobulin $\mathrm{M}$

\section{Introduction}

Asthma is a common chronic inflammatory disease of the airways, and is characterized by reversible airflow obstruction, bronchospasm and various recurring symptoms, including wheezing, chest tightness, coughing and shortness of breath (1). Furthermore, asthma affects both adults and children, and may occur in all populations and locations across the globe (2). Statistics showed that approximately 300 million people suffered from asthma worldwide, and approximately 250,000 annual mortalities are attributed to asthma, most of which are preventable (3). It has been reported that the prevalence of asthma in the population aged 0-17 years is 9.5\%, which is higher than that of adults aged 18 and over (7.7\%) for the period 2008-2010 in the United States (4). Additionally, research has also shown that $11 \%$ of 12 -year-old schoolchildren may suffer from severe asthma (4). Currently, there are no specific treatments for asthma and long-term standard treatment may reduce the recurrence of asthma, but evidence has also suggested that early intervention is essential for the patients with asthma (5-8). However, the diagnosis of asthma in children is difficult and easily misdiagnosed, which may result in under treatment of asthmatics or overtreatment of transient wheezes and lead to lung function impairment (9). Therefore, a correct and timely diagnosis of asthma in children is the first step towards effective management and treatment of asthma, which may reduce the potential harm due to misdiagnosis (10).

Previous findings showed that the presence of inflammation has become one of the most important markers in the diagnosis of asthma (11). Moreover, a variety of phenotypes of chronic asthma with persistent inflammation have been recognized, and a link between bacterial infections and refractory asthma has emerged $(12,13)$. Mycoplasma pneumoniae (MP), an extracellular pathogen lacking a cell wall, is a common causative agent of pneumonia in children and young adults (14). Most MP infections are limited to respiratory tract, with 3-10\% of patients developing clinical pneumonia; approximately $25 \%$ of patients with MP infections also suffer a variety of extra-pulmonary manifestations (15). Although the mechanism of MP pathogenesis remains to be elucidated, one of the known important components thereof is the induction of various cytokines and chemokines (16). Host immune factors may influence the outcome of infection, and previous findings showed that asthmatic children were lacking 
in cellular and humoral responses to MP infection $(17,18)$. MP may infect the upper and lower respiratory tract, causing pneumonia or bronchitis, and may be involved in the initiation and recurrence of asthma exacerbations $(19,20)$. It has been suggested that MP infection leads to changes of a variety of serum immune parameters, such as MP immunoglobulin M (MP-IgM), MP immunoglobulin E (MP-IgE), interleukin-18, eosinophils (EOS) count and others (21-23). Prior evidence has also shown that MP may link to new-onset asthma, exacerbations of asthma, chronic worsening of asthma and long-term decrements in pulmonary function $(24,25)$. However, defining a clear relationship between MP infection and asthma has been difficult, and the precise role and pathogenic mechanisms of MP in asthma risk are unclear.

In the current case-control investigation, we analyzed the serum-specific MP-IgM antibody levels and EOS count in asthmatic children in order to investigate the association between MP infection and the risk of asthma among children. Furthermore, we conducted a meta-analysis to test and verify the results of the case-control study.

\section{Materials and methods}

Ethics statement. The protocol of this study was carried out with the approval of the Institutional Review Board of Linyi People's Hospital (Shandong, China). Children were enrolled in this study after obtaining informed written consents from their parents. All the study procedures were in line with the Declaration of Helsinki (26).

Study participants. Between January 2013 and December 2014, a total of 139 children (87 males and 52 females; mean age, $6.65 \pm 2.28$ years; range, $0-15$ years) previously diagnosed with asthma were recruited from the Respiratory Care Department of Linyi People's Hospital and assigned into an experimental group. All the asthma patients were divided into three groups according to age: Group A (age $<3$ years, $n=42$ ); group B (age range, $3-8$ years, $n=45$ ) and group $C$ (age $>8$ years, $n=52$ ). In addition, 115 healthy children (68 males and 47 females; mean age, $6.39 \pm 2.34$ years; range, 1-12 years), without any history of respiratory tract infection, from the Medical Center were enrolled as the control group. The diagnosis of asthma was based on physician assessment according to the national guidelines published by the Subspecialty Group of Respiratory Diseases Society of Pediatrics of Chinese Medical Association in 2008 (27). The disease course of asthma in the patients was 6-20 days. The inclusion criteria were the following: i) Patients with recurring wheezing, short breath, chest distress or cough, which were mostly related to allergen contact, cold air, physical or chemical stimulation, viral respiratory tract infections and exercise; ii) patients that had wheezing breath sounds and a prolonged or interrupted expiratory flow; and iii) patients that had a notable curative effect on inhaled bronchodilators. Patients were excluded if they had severe heart disease, severe pulmonary disease and kidney disease, or had a history of allergic bronchial asthma. The diagnostic criteria of MP infection include: MP-IgM antibodies were positive; MP immunoglobulin G (MP-IgG)-negative antibodies changed into positive or the $\operatorname{IgG}$ antibody titers were elevated $>4$-fold (28).
Sample collection and detection (serum MP-IgM expression detection and EOS counting). Venous blood ( $2 \mathrm{ml})$ was drawn in the morning after an overnight fast at 7 th day of the disease course of asthma from all the children in the experimental and control groups. The collected samples were centrifuged at $1,500 \mathrm{x} g$ for $15 \mathrm{~min}$ to separate the serum. Enzyme-linked immunosorbent assay (ELISA) was used to measure the positive rate of MP-IgM in children, using a Serodia Myco II kit (Fujirebio Inc., Tokyo, Japan). Serum IgM level ( $>750 \mathrm{U} / \mathrm{ml}$ ) was considered to be a positive MP-IgM antibody level, suggesting MP infection. The EOS count was detected by a routine blood cell analyzer.

Treatments. Children with MP-IgM negative antibodies received $\beta$-lactam antibiotics (penicillin or cephalosporin). Patients with acute asthma were treated with bronchodilators or hormone therapy. Additionally to routine therapy, children with MP-IgM-positive antibodies received sequential therapy including $10 \mathrm{mg} / \mathrm{kg} / \mathrm{day}$ azithromycin. Patients took azithromycin continuously for 3-5 days until the body temperature dropped. After the clinical symptoms and signs were largely dissipated, patients were treated with an oral administration of $10 \mathrm{mg} / \mathrm{kg} /$ day azithromycin continuously for 3 days a week, over a course of 3 weeks. Blood and urine routine examinations, as well as liver function test were performed during the administration. When any unusual symptoms appeared, the medicine causing the effect was stopped.

Serum parameters of MP infection. The positive rate of MP-IgM and EOS count in the experimental and control groups were recorded. The comparison on the differences of MP-IgM-positive rate and EOS count between two groups was performed. The comparison on the differences of MP-IgM-positive rate and EOS count of asthma patients among the different age groups was performed.

Statistical analysis. Categorical data are expressed as numbers and percentages, and were analyzed using $\chi^{2}$ test. Continuous variables with normal distribution were expressed as mean \pm standard deviation (SD). The t-test was used to measure the differences between two groups of continuous variables. $\mathrm{P}<0.05$ was considered to indicate a statistically significant difference.

A comprehensive search was conducted for relevant studies published in PubMed (1966-2014), Cochrane Library (2005-2014), Wanfang database (1990-2014), Google Scholar and Chinese National Knowledge Infrastructure (1990-2014) databases using the following search terms: ('Mycoplasma pneumoniae' or 'MP') and ('asthma' or 'bronchial asthma') and ('child' or 'children'). The references of the eligible articles or textbooks were also reviewed to check through manual searches to identify other potential studies. Data from published studies were extracted independently by two authors to provide the necessary information. Any disagreement was resolved by discussion between the authors. A fixed or random effect model was used to measure the odds ratios (ORs) and its 95\% confidence intervals (CIs) to evaluate the associations between MP infection and asthma. The significance of the pooled estimate was made using the Z-test (29). Cochran's Q-statistic was applied to estimate the heterogeneity 
Table I. The demographic characteristics of the children with asthma and healthy controls.

\begin{tabular}{lccc}
\hline Groups & $\begin{array}{c}\text { Case group } \\
(\mathrm{n}=139)\end{array}$ & $\begin{array}{c}\text { Control group } \\
(\mathrm{n}=115)\end{array}$ & P-value \\
\hline Gender (M/F) & $87 / 52$ & $68 / 47$ & 0.574 \\
Age (years) & $6.65 \pm 2.28$ & $6.39 \pm 2.34$ & 0.372 \\
BMI (kg/cm $\left.{ }^{2}\right)$ & $19.8 \pm 2.1$ & $20.2 \pm 2.5$ & 0.167 \\
ETS, $\mathrm{n}(\%)$ & $91(65.5 \%)$ & $66(57.4 \%)$ & 0.187 \\
Family history of & $20(14.4 \%)$ & $8(7.0 \%)$ & 0.060 \\
asthma, $\mathrm{n}(\%)$ & & & \\
Urban family, $\mathrm{n}(\%)$ & $85(61.2 \%)$ & $62(53.9 \%)$ & 0.245 \\
$\begin{array}{l}\text { Exposure to dust } \\
\text { environment, n }(\%)\end{array}$ & $33(23.7 \%)$ & $24(20.9 \%)$ & 0.418 \\
\hline
\end{tabular}

M, male; F, female; BMI, body mass index; ETS, environmental tobacco smoke.

among studies, and $\mathrm{P}<0.05$ was considered to be statistically significant (30). The $\mathrm{I}^{2}$ test was also used to quantify the heterogeneity (range from 0 to $100 \%$ ) (31). Random-effect model (DerSimonian-Laird method) was used when $\mathrm{P}<0.05$ or $\mathrm{I}^{2}>50 \%$. Fixed-effects model (Mantel-Haenszel method) was used when there was no statistical heterogeneity (32). Data for the meta-analysis were analyzed by the Comprehensive MetaAnalysis version 2.0 software (Biostat Inc., Englewood, NJ, USA). Begg's funnel plot and Egger's test were performed to assess the publication bias of articles in all comparison models.

\section{Results}

Baseline characteristics of study population. Table I shows the baseline characteristics of subjects in the experimental and control groups. No significant difference was observed in gender, age, body mass index (BMI), and exposure to environmental tobacco smoke (ETS) between children with asthma and healthy controls (all $\mathrm{P}>0.05$ ). Children with asthma had a higher rate of family history of asthma when compared with the control group, but with no significant difference (14.4 vs. 7.0\%, $\mathrm{P}=0.060$ ). Furthermore, the rates of children in urban families and the rate of exposure to dust in the experimental group were higher than those in the control group, but with no significant difference (urban family: 61.2 vs. $53.9 \%, \mathrm{P}=0.245$; exposure to dust: 23.7 vs. $20.9 \%, \mathrm{P}=0.418)$.

Positive rate of serum IgM antibodies. Of all subjects, 54.7\% (76/139) of asthma patients and $15.7 \%(18 / 115)$ of the control group were found to be positive for MP-IgM antibodies. The positive rate of MP-IgM in the experimental group was significantly higher than that in the control group (54.7 vs. $15.7 \%$, $\chi^{2} 41.11, \mathrm{P}<0.001$ ) (Fig. 1). ELISA results showed that 17 (40.5\%) patients in group A, 22 (48.9\%) patients in group B and $37(71.2 \%)$ patients in group $\mathrm{C}$ were detected to be MP-IgM antibody-positive. There was no significant difference in the positive rate of MP-IgM between groups A and B (40.5 vs. $\left.48.9 \%, \chi^{2} 0.62, \mathrm{P}=0.430\right)$. However, the positive rates of MP-IgM in groups A and B were significantly lower

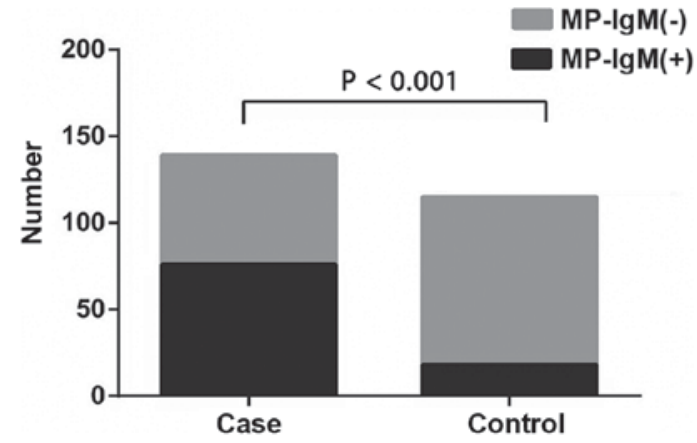

Figure 1. The positive rates of MP-IgM in asthmatic children (case group) and healthy controls (control group). The MP-IgM-positive rate in asthmatic children was significantly higher than that in the healthy controls $(\mathrm{P}<0.001)$. MP-IgM, Mycoplasma pneumoniae immunoglobulin M.

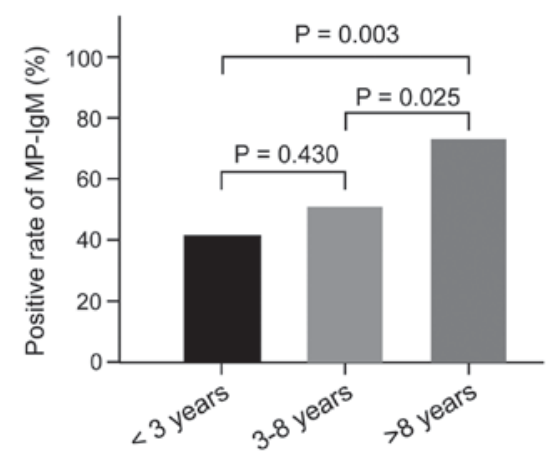

Figure 2. The positive rates of MP-IgM of asthmatic children in different age groups. The asthmatic children aged more than 8 years had a higher positive rate of MP-IgM than the asthmatic children aged $<3$ years, and the asthmatic children aged 3-8 years, respectively. MP-IgM, Mycoplasma pneumoniae immunoglobulin M.

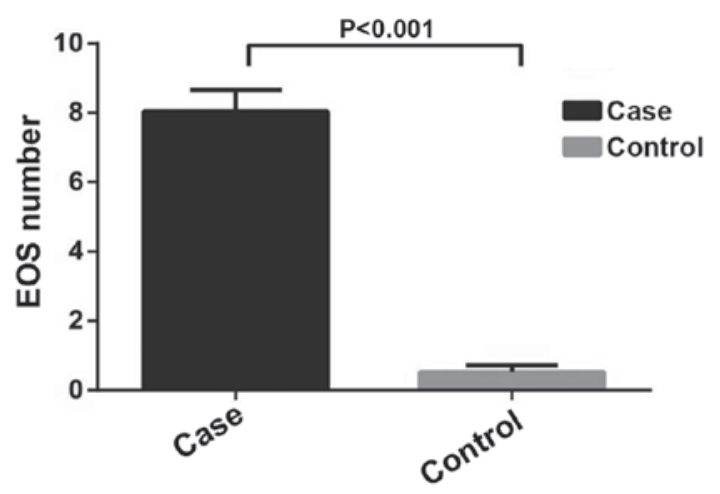

Figure 3. The EOS count in asthmatic children (case group) and healthy controls (control group). The EOS count in asthmatic children was significantly higher than that in the healthy controls $(\mathrm{P}<0.001)$. EOS, eosinophils.

than those in group C, respectively (group A vs. group C: 40.5 vs. $71.2 \%, \chi^{2}=8.95, P=0.003$; group $\mathrm{B}$ vs. group $\mathrm{C}$ : 48.9 vs. $71.2 \%, \chi^{2}=5.02, \mathrm{P}=0.025$ ) (Fig. 2).

Association of EOS count with asthma risk. The results showed that the blood EOS count in asthma patients was markedly higher than those in healthy controls $\left(8.04 \pm 0.6210^{9} / 1\right.$ vs. $0.52 \pm 0.2010^{9} / 1, \mathrm{t}=124.8, \mathrm{P}<0.001$ ) (Fig. 3). According to the different age groups of asthma patients, we found that the 


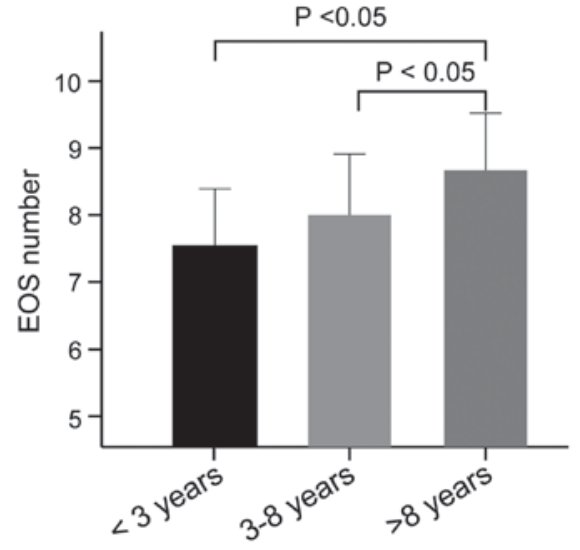

Figure 4. The EOS count of asthmatic children in different age groups. The asthmatic children aged more than 8 years had an increased EOS count as compared to that in the asthmatic children aged $<3$ years and the asthmatic children aged 3-8 years, respectively. EOS, eosinophils. $\mathrm{P}<0.05$.

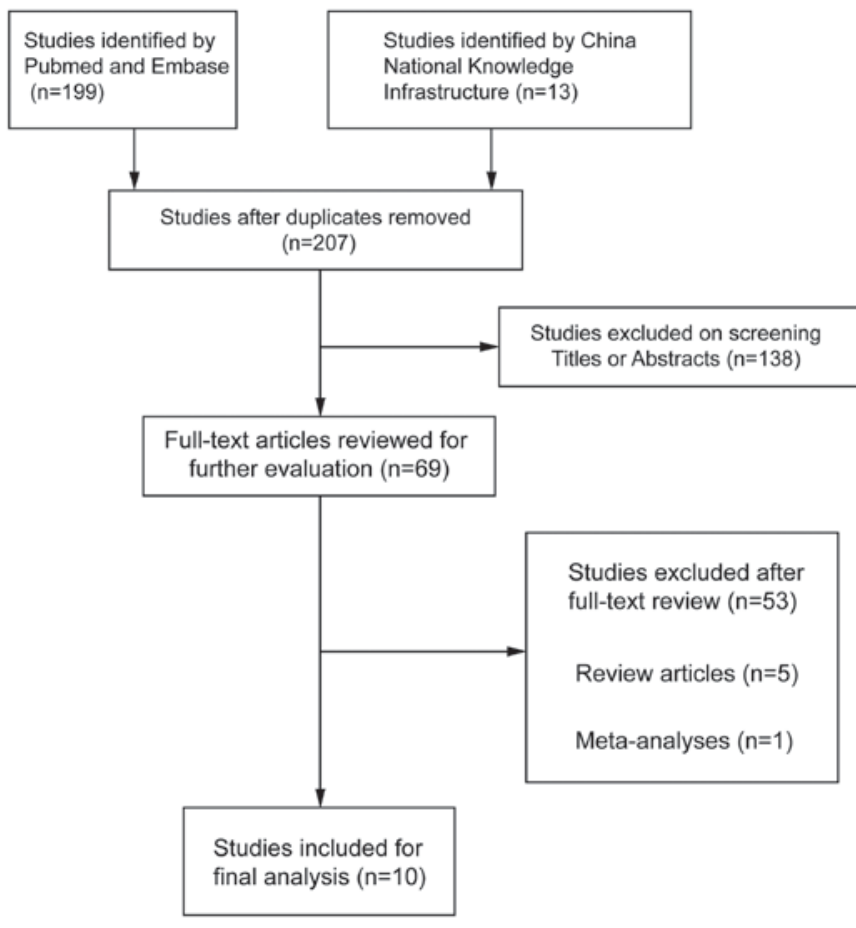

Figure 5. Flow diagram of the studies identified.

blood EOS count in groups A, B and C was $(7.56 \pm 1.62) 10^{9} / 1$, $(8.01 \pm 1.32) 10^{9} / 1$ and $(8.65 \pm 1.09) 10^{9} / 1$, respectively. No significant difference in the blood EOS count was observed between groups $\mathrm{A}$ and $\mathrm{B}(\mathrm{t}=1.43, \mathrm{P}=0.158)$. Nevertheless, the blood EOS count in groups $\mathrm{A}$ and $\mathrm{B}$ were significantly lower than that in group $\mathrm{C}$, respectively (group A vs. C: $\mathrm{t}=3.89, \mathrm{P}<0.05$; group B vs. $\mathrm{C}$ : $\mathrm{t}=2.62, \mathrm{P}<0.05$ ) (Fig. 4).

Results of meta-analysis. A total of 207 articles associated with the searched keywords were identified. Of these studies, 138 were excluded based on the titles and abstracts; another 59 studies were eliminated after reviewing the full text. Eventually, 10 studies met our inclusion criteria for this meta-analysis (25,33-41): H.-E. Zeng-Hong, Correlation Between Mycoplasma Pnenumonia Infection and Pediatric

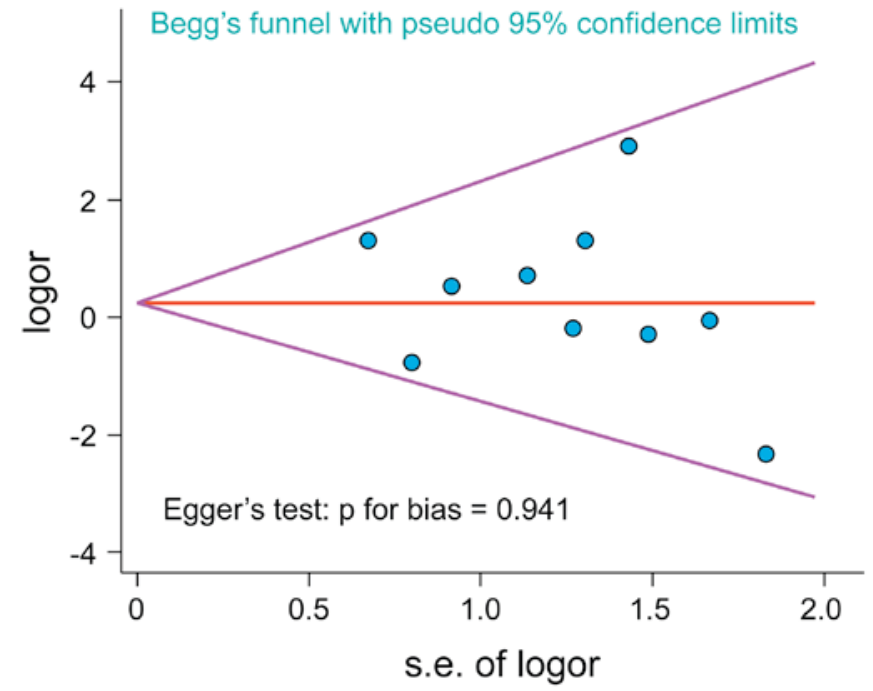

Figure 6. Publication bias were evaluated by Begg's funnel plot and Egger's test. The shape of the funnel plots were symmetrical and P-value for Egger's test was 0.941 , suggesting that there was no evidence of publication bias among these 10 included studies.

Asthma, 2011; Jiang Hua, Mycoplasma pneumoniae Infection and Bronchial Asthma in Children, 2013; Zhao Zhi-Yun, Observation on Infection Results and Correlation Between Childhood Asthma and Mycoplasma pneumoniae, 2014; Liu Chunxiang, Association of Mycoplasma pneumoniae Infection and Pediatric Asthma, 2009) (Fig. 5). Egger's test and Begg's funnel plot were used to statistically assess the funnel plot symmetry. The results suggested no evidence of publication bias for the included 10 studies (Fig. 6). These 10 case-control studies were composed of 795 asthma patients and 630 healthy controls. Publication year of articles included ranged from 2000 to 2014 . There was significant heterogeneity observed $\left(\mathrm{I}^{2}=63.95 \%, \mathrm{P}_{\mathrm{h}}<0.05\right)$; thereby the random effects model was carried out. Meta-analysis results showed that the MP-IgM-positive rate in asthma patients were markedly higher than that of healthy controls $(\mathrm{OR}=3.496,95 \% \mathrm{CI}=2.060-5.933$, $\mathrm{P}<0.001$ ) (Fig. 7). Age-stratified analysis presented that the MP-IgM-positive rate in both children $\geq 8$ and $<8$ years were significantly higher than that of healthy controls (average age, $\geq 8$ years: $\mathrm{OR}=3.074,95 \% \mathrm{CI}=1.455-6.494, \mathrm{P}=0.003$; average age, $<8$ years: $\mathrm{OR}=4.762,95 \% \mathrm{CI}=2.570-8.822$, $\mathrm{P}<0.001)$ (Fig. 8).

\section{Discussion}

MP is a highly specialized bacterium that functions as an obligate parasite in the respiratory tract of humans, which may be involved in establishing a chronic infection (36). Furthermore, research has shown that MP infects the upper and lower respiratory tract which may be associated with the occurrence of bronchitis and asthma; however, the underlying mechanism is not completely understood $(42,43)$. In this regard, we performed a case-control study and a meta-analysis to explore the correlation of MP infection with the increased risk of asthma. This study found that MP-IgM-positive rate was higher in asthmatic children when compared with healthy controls, indicating that elevated MP-IgM-positive rate may 


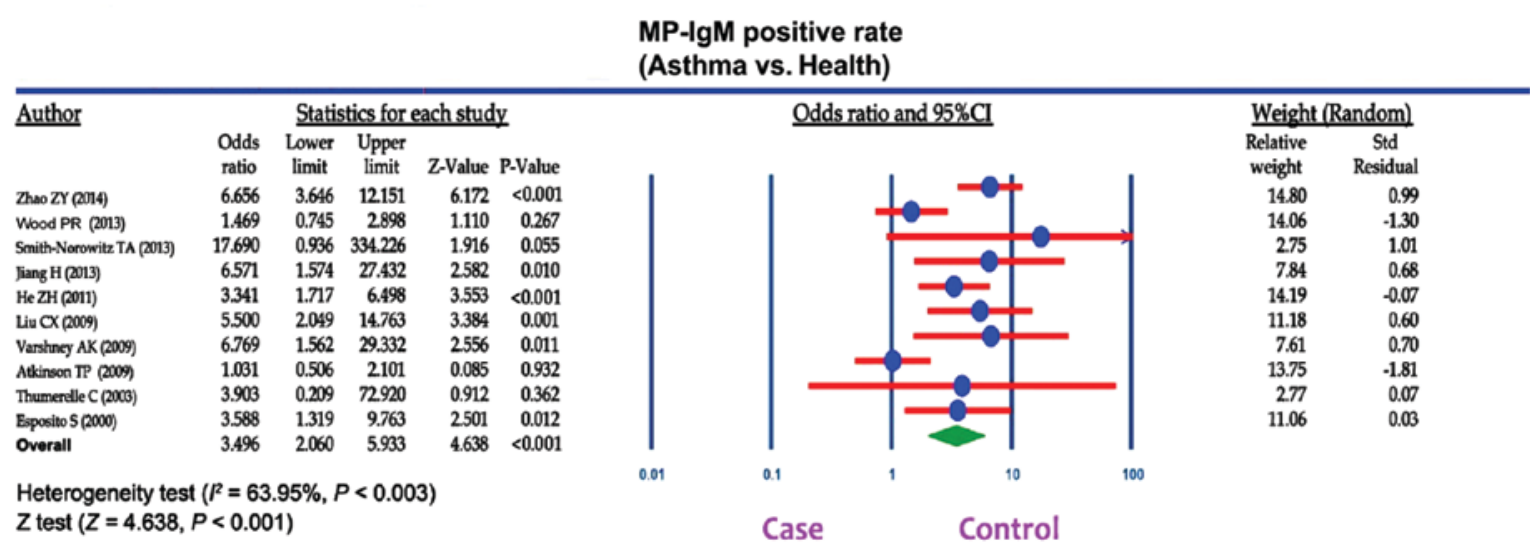

Figure 7. Forest plots of relationship of Mycoplasma pneumoniae immunoglobulin M (MP-IgM)-positive rate with the risk of asthma among children.

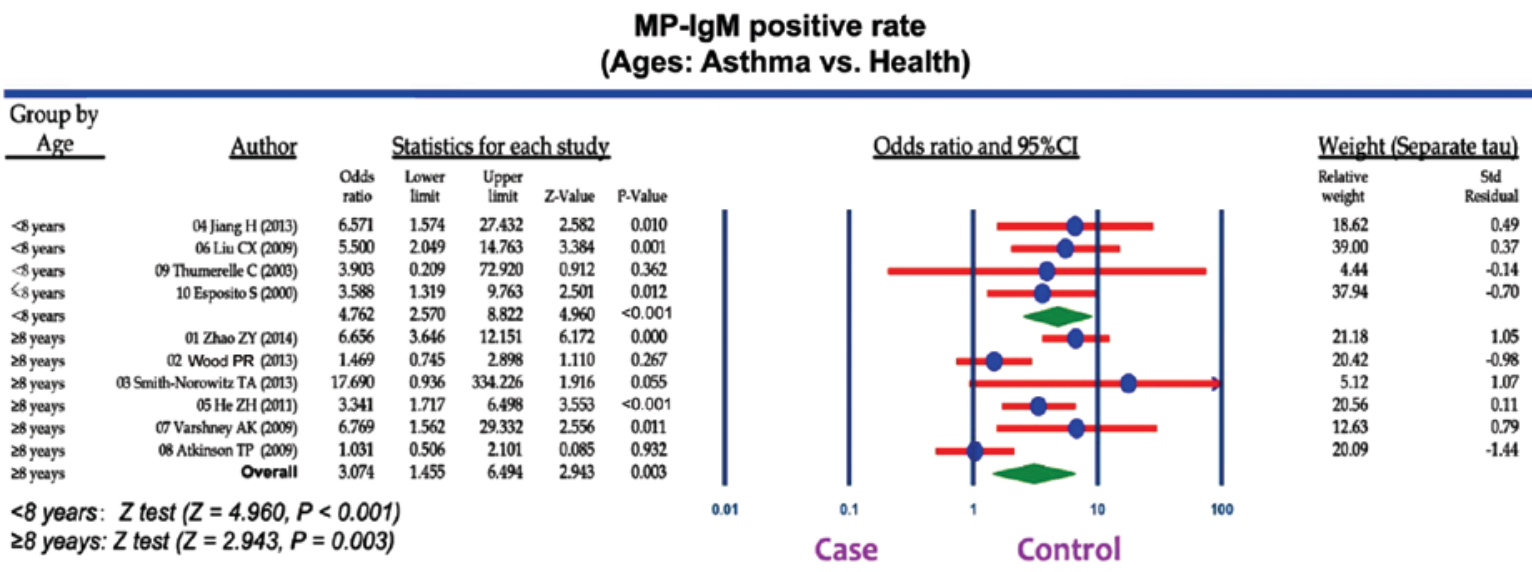

Figure 8. Stratified analysis for the difference of Mycoplasma pneumoniae immunoglobulin M (MP-IgM)-positive rate between the asthmatic children (case group) and the healthy controls (control group).

be involved in the development of asthma among children. Generally, the elevated MP-IgM-positive rate can be used as an early diagnostic criterion of the initial acute MP infection (44). As a specific antigen, MP can induce immediate or delayed hypersensitivity reaction, and may result in allergic airway inflammation and induce the production of $\operatorname{IgE}$ and IgM, which may mediate type 1 allergic reaction. Therefore, MP may lead to the development of asthma (45). Additionally, MP infection is associated with significant specific IgM responses in addition to specific IgG and IgE responses; thus, the production of specific MP-IgM may have a role in the exacerbation of asthma (17,46). High positive levels of MP-IgM antibodies were found in patients who were infected with MP during asthma exacerbation (37). We suspected that the possible explanations of how MP affects the airway included the production of specific MP-IgM antibodies in addition to direct effects on airway epithelium, inflammatory reaction on airways and alteration in the autonomic nervous system (36). Smith-Norowitz et al found that the IgM-positivity was apparently higher in asthmatic patients as compared to the healthy controls, and suggested that the increased specific MP-IgM responses may play an important role in MP infection in asthma development (35). Previous report by Varshney et al highlighted that MP may play a crucial role in the onset of asthma in predisposed children and be a trigger for recurrent wheezing (37). Consistent with previous studies, our study also demonstrated that the MP infection accompanied with higher MP-IgM-positive rate may be involved in the development of asthma. Additionally, we conducted a meta-analysis to confirm the associations and found that the asthmatic children had a higher MP-IgM-positive rate, indicating that a higher MP-IgM-positive rate may be associated with an increased susceptibility to asthma.

Another important finding in our study revealed that the levels of EOS in asthmatic children were significantly higher than those in the non-asthmatic controls. This leads us to believe that patients with asthma may suffer from allergy inflammation and the elevated EOS count may be crucial in the pathogenesis of asthma. EOS is thought to have a key pro-inflammatory role in the pathogenesis of asthma as its mediators are identified in asthmatic, but not healthy lungs. The suppression of eosinophil infiltration in various clinical diseases by glucocorticoids may be correlated with an amelioration of symptoms and disordered airway function (47). Characterized as an eosinophilic airway inflammation, asthma is often considered to be associated with a higher peripheral blood EOS count, and the EOS may play an important role in the pathogenesis of bronchial asthma $(48,49)$. Additionally, airway hyper-responsiveness has been reported to be closely correlated with the presence of EOS $(50,51)$. Early studies found that the respiratory tract infections with a 
higher EOS count had a higher risk of asthma when compared with the respiratory tract infectors with normal EOS count, suggesting that the EOS count is an important clinical index in predicting the development of asthma $(52,53)$. Furthermore, we found that the asthmatic children aged $>8$ years had higher MP-IgM-positive rate and EOS count as compared to the asthmatic children aged $<3$ years and asthmatic children aged 3-8 years. We suspected that these differences may be correlated with the clinically significant alterations in lung function. However, the underlying mechanism involved remains unclear; therefore, further studies based on the involvement of mechanistic pathways in asthma in children of different ages should be conducted.

In conclusion, the present study suggests that asthmatic children have a higher MP-IgM-positive rate and EOS count when compared with non-asthmatic controls, indicating that MP infection may promote the production of $\operatorname{IgM}$ in asthmatic children. Deficient host defense mechanisms in children with asthma may contribute to a higher prevalence of MP-IgM, which likely reflects an increased susceptibility to asthma. However, with our own limitations, such as small sample size and the inclusion and exclusion criterion of the meta-analysis may affect the statistical power of this study. Future large-scale prospective studies are needed to confirm the correlations and predictive value of MP infection with asthma risk.

\section{Acknowledgements}

We would like to acknowledge the reviewers for their helpful comments on this study.

\section{References}

1. Urbano FL: Review of the NAEPP 2007 Expert Panel Report (EPR-3) on Asthma Diagnosis and Treatment Guidelines. J Manag Care Pharm 14: 41-49, 2008.

2. Centers for Disease Control and Prevention (CDC): Vital signs: asthma prevalence, disease characteristics, and self-management education: United States, 2001 - 2009. MMWR Morb Mortal Wkly Rep 60: 547-552, 2011.

3. Croisant S: Epidemiology of asthma: prevalence and burden of disease. Adv Exp Med Biol 795: 17-29, 2014.

4. Akinbami LJ, Moorman JE, Bailey C, Zahran HS, King M, Johnson CA and Liu X: Trends in asthma prevalence, health care use, and mortality in the United States, 2001-2010. NCHS Data Brief: 1-8, 2012.

5. Holgate ST: A look at the pathogenesis of asthma: the need for a change in direction. Discov Med 9: 439-447, 2010.

6. Corren J, Lemanske RF Jr, Hanania NA, Korenblat PE, Parsey MV, Arron JR, Harris JM, Scheerens H, Wu LC, Su Z, et al: Lebrikizumab treatment in adults with asthma. N Engl J Med 365: 1088-1098, 2011.

7. Hafkamp-de Groen E, Mohangoo AD, de Jongste JC, van der Wouden JC, Moll HA, Jaddoe VW, Hofman A, de Koning $\mathrm{HJ}$ and Raat $\mathrm{H}$ : Early detection and counselling intervention of asthma symptoms in preschool children: study design of a cluster randomised controlled trial. BMC Public Health 10 $555,2010$.

8. National Asthma Education and Prevention Program: Expert Panel Report 3 (EPR-3): Guidelines for the Diagnosis and Management of Asthma-Summary Report 2007. J Allergy Clin Immunol 120 (Suppl 5): S94-S138, 2007.

9. Cave AJ and Atkinson LL: Asthma in preschool children: a review of the diagnostic challenges. J Am Board Fam Med 27 538-548, 2014.

10. Busse WW: Asthma diagnosis and treatment: filling in the information gaps. J Allergy Clin Immunol 128: 740-750, 2011.

11. Dahlén SE, Dahlén B and Drazen JM: Asthma treatment guidelines meet the real world. N Engl J Med 364: 1769-1770, 2011
12. Locksley RM: Asthma and allergic inflammation. Cell 140: 777-783, 2010.

13. Busse WW: The relationship of airway hyperresponsiveness and airway inflammation: Airway hyperresponsiveness in asthma: its measurement and clinical significance. Chest 138 (Suppl 2): 4S-10S, 2010.

14. Bébéar C, Pereyre S and Peuchant O: Mycoplasma pneumoniae: susceptibility and resistance to antibiotics. Future Microbiol 6: 423-431, 2011.

15. Khan FY and Sayed H: Rhabdomyolysis associated with Mycoplasma pneumoniae pneumonia. Hong Kong Med J 18: 247-249, 2012.

16. van der Poll T and Opal SM: Pathogenesis, treatment, and prevention of pneumococcal pneumonia. Lancet 374 : 1543-1556, 2009

17. Waites KB, Balish MF and Atkinson TP: New insights into the pathogenesis and detection of Mycoplasma pneumoniae infections. Future Microbiol 3: 635-648, 2008.

18. Narita M: Pathogenesis of extrapulmonary manifestations of Mycoplasma pneumoniae infection with special reference to pneumonia. J Infect Chemother 16: 162-169, 2010.

19. Hong SJ: The role of Mycoplasma pneumoniae infection in asthma. Allergy Asthma Immunol Res 4: 59-61, 2012.

20. Watanabe H, Uruma T, Nakamura $\mathrm{H}$ and Aoshiba K: The role of Mycoplasma pneumoniae infection in the initial onset and exacerbations of asthma. Allergy Asthma Proc 35: 204-210, 2014.

21. Wang M, Wang Y, Yan Y, Zhu C, Huang L, Shao X, Xu J, Zhu H, Sun X, Ji W, et al: Clinical and laboratory profiles of refractory Mycoplasma pneumoniae pneumonia in children. Int J Infect Dis 29: 18-23, 2014.

22. Wenzel S, Ford L, Pearlman D, Spector S, Sher L, Skobieranda F, Wang L, Kirkesseli S, Rocklin R, Bock B, et al: Dupilumab in persistent asthma with elevated eosinophil levels. N Engl J Med 368: 2455-2466, 2013

23. Schleich FN, Seidel L, Sele J, Manise M, Quaedvlieg V, Michils A and Louis R: Exhaled nitric oxide thresholds associated with a sputum eosinophil count $\geq 3 \%$ in a cohort of unselected patients with asthma. Thorax 65: 1039-1044, 2010.

24. Atkinson TP and Waites KB: Mycoplasma pneumoniae infections in childhood. Pediatr Infect Dis J 33: 92-94, 2014.

25. Wood PR, Hill VL, Burks ML, Peters JI, Singh H, Kannan TR, Vale S, Cagle MP, Principe MF, Baseman JB, et al: Mycoplasma pneumoniae in children with acute and refractory asthma. Ann Allergy Asthma Immunol 110: 328-334, 2013.

26. Holt GR: Declaration of Helsinki - the world's document of conscience and responsibility. South Med J 107: 407, 2014.

27. Subspecialty Group of Respiratory Diseases Society of Pediatrics; Chinese Medical Association; Chinese Journal of Pediatrics Editorial Board: Guideline for the diagnosis and optimal management of asthma in children. Zhonghua Er Ke $\mathrm{Za}$ Zhi 46: 745-753, 2008 (In Chinese).

28. Zhang YM and Liu XY: Advance in the diagnosis and treatment of Mycoplasma pneumoniae pneumonia and related complications. Zhongguo Dang Dai Er Ke Za Zhi 13: 358-360, 2011 (In Chinese).

29. Chen H, Manning AK and Dupuis J: A method of moments estimator for random effect multivariate meta-analysis. Biometrics 68: 1278-1284, 2012.

30. Jackson D, White IR and Riley RD: Quantifying the impact of between-study heterogeneity in multivariate meta-analyses. Stat Med 31: 3805-3820, 2012.

31. Peters JL, Sutton AJ, Jones DR, Abrams KR and Rushton L: Comparison of two methods to detect publication bias in meta-analysis. JAMA 295: 676-680, 2006.

32. Zintzaras E and Ioannidis JP: Heterogeneity testing in meta-analysis of genome searches. Genet Epidemiol 28: $123-137,2005$

33. Atkinson TP, Duffy LB, Pendley D, Dai Y and Cassell GH: Deficient immune response to Mycoplasma pneumoniae in childhood asthma. Allergy Asthma Proc 30: 158-165, 2009.

34. Esposito S, Blasi F, Arosio C, Fioravanti L, Fagetti L, Droghetti R, Tarsia P, Allegra L and Principi N: Importance of acute Mycoplasma pneumoniae and Chlamydia pneumoniae infections in children with wheezing. Eur Respir J 16: 1142-1146, 2000.

35. Smith-Norowitz TA, Silverberg JI, Kusonruksa M, Weaver D, Ginsburg D, Norowitz KB, Durkin HG, Hammerschlag MR, Bluth MH and Kohlhoff SA: Asthmatic children have increased specific anti-Mycoplasma pneumoniae IgM but not IgG or IgE-values independent of history of respiratory tract infection. Pediatr Infect Dis J 32: 599-603, 2013. 
36. Thumerelle C, Deschildre A, Bouquillon C, Santos C, Sardet A, Scalbert M, Delbecque L, Debray P, Dewilde A, Turck D, et al: Role of viruses and atypical bacteria in exacerbations of asthma in hospitalized children: a prospective study in the Nord-Pas de Calais region (France). Pediatr Pulmonol 35: 75-82, 2003.

37. Varshney AK, Chaudhry R, Saharan S, Kabra SK, Dhawan B, Dar L and Malhotra P: Association of Mycoplasma pneumoniae and asthma among Indian children. FEMS Immunol Med Microbiol 56: 25-31, 2009.

38. He ZH, Zhang JT and Sun YF: Correlation between mycoplasma pnenumonia infection and pediatric asthma. Chin J Nosocomiol 21: 4733-4734, 2011 (In Chinese).

39. Jiang H: Mycoplasma pneumoniae infection and bronchial asthma in children. Chin J Med Guide 15: 1275-1276, 2013 (In Chinese)

40. Liu CX: Analysis of the relationship between bronchial asthma and Mycoplasma pneumoniae infection. Chin J Postgrad Med 32: 49-50, 2009 (In Chinese). http://d.g.wanfangdata.com. cn/Periodical_ysjxzz200915022.aspx.

41. Zhao ZY: Observation on infection results and correlation between childhood asthma and Mycoplasma pneumoniae. J Clin Pulm Med 8: 1497-1498,1522, 2014 (In Chinese).

42. Guilbert TW and Denlinger LC: Role of infection in the development and exacerbation of asthma. Expert Rev Respir Med 4 71-83, 2010.

43. Rollins DR, Beuther DA and Martin RJ: Update on infection and antibiotics in asthma. Curr Allergy Asthma Rep 10 67-73, 2010.

44. Ke LQ, Wang FM, Li YJ and Luo YC: Epidemiological characteristics of Mycoplasma pneumoniae pneumonia in children. Zhongguo Dang Dai Er Ke Za Zhi 15: 33-36, 2013 (In Chinese).
45. Burton OT and Oettgen HC: Beyond immediate hypersensitivity: evolving roles for IgE antibodies in immune homeostasis and allergic diseases. Immunol Rev 242: 128-143, 2011.

46. Defilippi A, Silvestri M, Tacchella A, Giacchino R, Melioli G, Di Marco E, Cirillo C, Di Pietro P and Rossi GA: Epidemiology and clinical features of Mycoplasma pneumoniae infection in children. Respir Med 102: 1762-1768, 2008.

47. Green RH, Brightling CE, McKenna S, Hargadon B, Parker D, Bradding P, Wardlaw AJ and Pavord ID: Asthma exacerbations and sputum eosinophil counts: a randomised controlled trial. Lancet 360: 1715-1721, 2002.

48. Fleming L, Wilson N, Regamey N and Bush A: Use of sputum eosinophil counts to guide management in children with severe asthma. Thorax 67: 193-198, 2012.

49. Nakagome K and Nagata M: Pathogenesis of airway inflammation in bronchial asthma. Auris Nasus Larynx 38: 555-563, 2011.

50. van Rensen EL, Evertse CE, van Schadewijk WA, van Wijngaarden S, Ayre G, Mauad T, Hiemstra PS, Sterk PJ and Rabe KF: Eosinophils in bronchial mucosa of asthmatics after allergen challenge: effect of anti-IgE treatment. Allergy 64 : 72-80, 2009.

51. Tantisira KG, Colvin R, Tonascia J, Strunk RC, Weiss ST and Fuhlbrigge AL; Childhood Asthma Management Program Research Group: Airway responsiveness in mild to moderate childhood asthma: sex influences on the natural history. Am J Respir Crit Care Med 178: 325-331, 2008

52. Apter AJ: Advances in adult asthma diagnosis and treatment and HEDQ in 2010. J Allergy Clin Immunol 127: 116-122, 2011

53. Laviolette M, Gossage DL, Gauvreau G, Leigh R, Olivenstein R, Katial R, Busse WW, Wenzel S, Wu Y, Datta V, et al: Effects of benralizumab on airway eosinophils in asthmatic patients with sputum eosinophilia. J Allergy Clin Immunol 132: 1086-1096, 2013. 